Supporting Information

for

\title{
Robust Nanoporous Membranes Templated by a Doubly Reactive Block Copolymer
}

\author{
Liang Chen, ${ }^{\dagger}$ William A. Phillip, ${ }^{*}$ E. L. Cussler, ${ }^{*}$ Marc A. Hillmyer ${ }^{*}{ }^{\dagger}$ \\ ${ }^{\dagger}$ Department of Chemistry, University of Minnesota, Minneapolis, MN 55455 \\ ${ }^{\ddagger}$ Department of Chemical Engineering \& Materials Science, University of Minnesota, Minneapolis, MN 55455
}




\section{Synthetic details.}

Hydroxyl group terminated polylactide (PLA) was prepared via aluminum-catalyzed ring-opening polymerization of D,L-lactide, and PLA-attached trithiocarbonate as a chain transfer agent (PLA-CTA) was synthesized following a reported procedure. ${ }^{1}$ Synthesis of a PLA- $b-\mathrm{P}(\mathrm{N}-s-\mathrm{S})$ diblock copolymer by reversible addition-fragmentation chain transfer (RAFT) polymerization is described as follows: PLA-CTA $\left(0.45 \mathrm{~g}, \mathrm{M}_{\mathrm{n}}\left({ }^{1} \mathrm{H} \mathrm{NMR}\right)=34 \mathrm{~kg} \mathrm{~mol}^{-1}, \mathrm{M}_{\mathrm{n}}(\mathrm{SEC})=40 \mathrm{~kg} \mathrm{~mol}^{-1}\right.$, polydispersity index (PDI) = 1.26), $p$-norbornenylethyl styrene ${ }^{2}(\mathrm{~N})(1.1 \mathrm{~mL})$, styrene $(1.1 \mathrm{~mL})$, and 2,2'-Azoisobutyronitrile (AIBN) $(0.21 \mathrm{mg})$ were dissolved in dry toluene $(1.3 \mathrm{~mL})$ in an air-free flask, and after three freeze-pump-thaw cycles the mixture was reacted at $70 \pm 1{ }^{\circ} \mathrm{C}$ for $20 \mathrm{~h}$. Copolymers were recovered through precipitation in methanol, followed by dissolution in $\mathrm{CH}_{2} \mathrm{Cl}_{2}$, reprecipitation in pentane, and drying under vacuum $(0.65 \mathrm{~g}$, $\left.\mathrm{M}_{\mathrm{n}}\left({ }^{1} \mathrm{H} \mathrm{NMR}\right)=50 \mathrm{~kg} \mathrm{~mol}^{-1}, \mathrm{M}_{\mathrm{n}}(\mathrm{SEC})=46 \mathrm{~kg} \mathrm{~mol}^{-1}, \mathrm{PDI}=1.40\right)$. A calculated monomer conversion was only $12 \mathrm{wt} \%$ under this condition, which was critical to minimize radical-based coupling reaction on norbornene groups. PLA- $b$-P(N-s-S) (0.2 g), dicyclopentadiene (DCPD) (0.1 g), and THF (0.9 mL) were pre-mixed in a glass vial with stirring and added to a $2^{\text {nd }}$ generation Grubbs catalyst $(1.5 \mathrm{mg})$ solution of THF $(0.15 \mathrm{~mL})$. Subsequently, the solution was casted on a silica or glass substrate, crosslinked at room temperature for $6 \mathrm{~h}$ with slow THF evaporation, and annealed at $100{ }^{\circ} \mathrm{C}$ for $1 \mathrm{~h}$ under air. PLA block in membranes was degraded in $0.5 \mathrm{M} \mathrm{NaOH}$ solution of a 40/60 (v/v) mixture of $\mathrm{MeOH}$ and $\mathrm{H}_{2} \mathrm{O}$ at $70{ }^{\circ} \mathrm{C}$ for 3 days. Finally, the resulted membranes were rinsed with $\mathrm{MeOH}$ and dried under vacuum at $50{ }^{\circ} \mathrm{C}$.

\section{Measurements.}

Size-exclusion chromatography (SEC) data were collected using a Hewlett-Packard 1100 series liquid chromatograph equipped with Jordi polydivinylbenzene columns with pore sizes of 10000, 1000, and 500 $\AA ̊$ as well as a Hewlett-Packard 1047A refractive index detector. THF was used as the mobile phase at 40 ${ }^{\circ} \mathrm{C}$ with a flow rate of $1 \mathrm{~mL} / \mathrm{min}$. The instrument was calibrated with polystyrene standards (Polymer Laboratories). Scanning electron microscopy (SEM) was performed on a Hitachi S-900 FE-SEM using $3.0 \mathrm{kV}$ accelerating voltage. Before SEM analysis, freeze-fractured polymer membranes were coated with a $1.5 \mathrm{~nm}$ thick Pt layer by direct Pt sputtering. Transmission electron microscopy (TEM) analysis was

\footnotetext{
${ }_{1}^{1}$ Rzayev, J.; Hillmyer, M. A. Macromolecules 2005, 38, 3-5.
}

${ }^{2}$ Chen, L.; Hillmyer, M. A. in preparation. 
conducted on a JOEL 1210 transmission electron microscope. Crosslinked membranes were microtomed at RT and stained with $\mathrm{OsO}_{4}$ vapor for $15 \mathrm{~min}$. FT-IR spectra were recorded on a Nicolet Magna-Infrared Spectrometer 550, and all samples were ground with $\mathrm{KBr}$ and pressed into thin sheets. Thermogravimetric analysis (TGA) was performed on a Perkin-Elmer TGA 7 instrument under air. The $\mathrm{N}_{2}$ adsorption experiment was carried out on a Micromeritics ASAP 2000 instrument. Tensile tests were measured using a Rheometric Scientific MINIMAT instrument at RT with a 1000 N load cell installed. Membranes before and after degradation were cut into a dogbone shape (1.0 $\mathrm{cm}$ in length). SAXS experiments in Figure S2 were conducted at the Argonne National Labs using the Advanced Photon Source, and other SAXS measurements were performed on a 2.3 m custom built beam line at the University of Minnesota. CuKa X-rays $(\lambda=1.542 \AA)$ were generated through the use of a rotating anode. Samples were equilibrated under vacuum for $300 \mathrm{~s}$ before collection. The 2-D images were azimuthally integrated to a 1-D plot of intensity versus $q$ where $q=4 \pi / \lambda \sin (\theta / 2)$ and $\theta$ and $\lambda$ are the scattering angle and X-ray wavelength, respectively. 


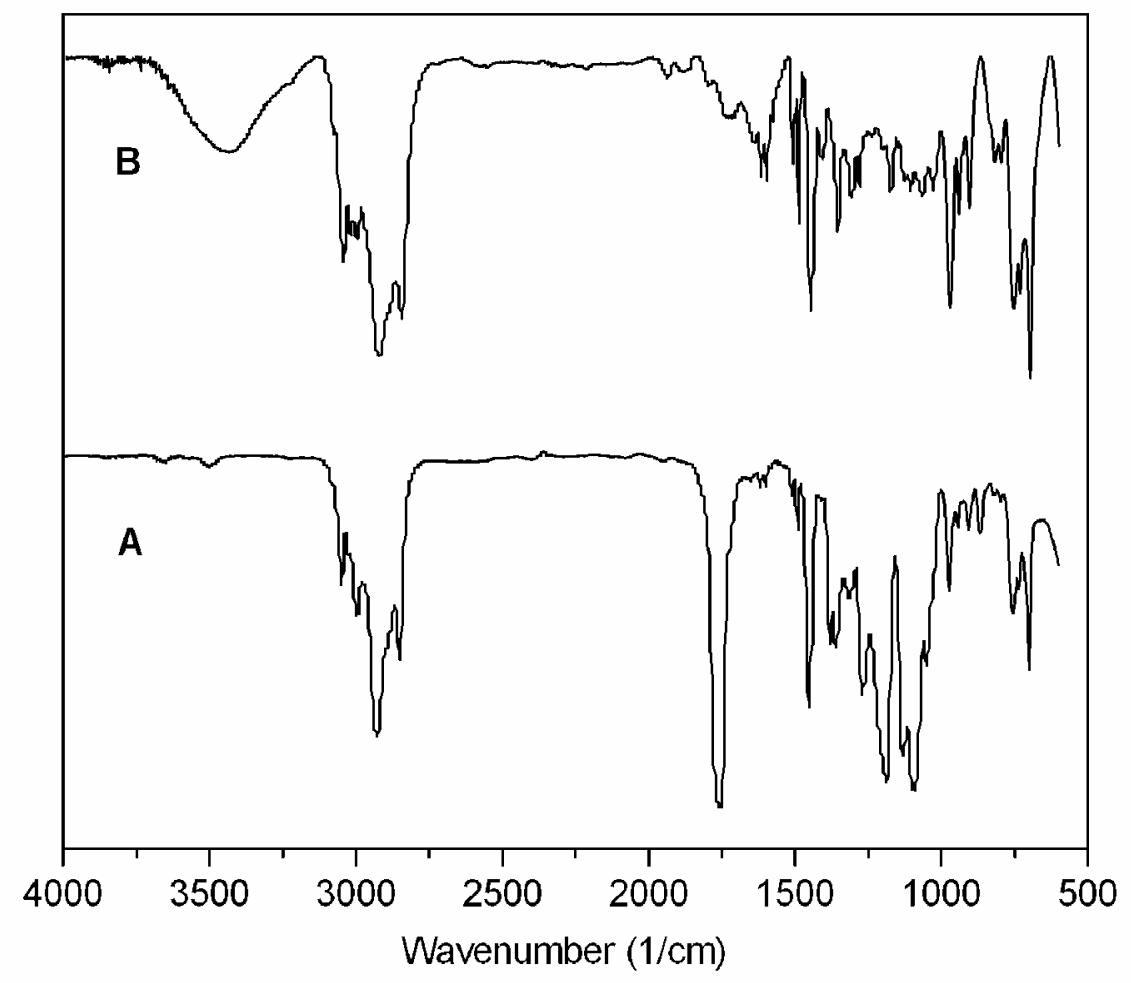

Figure S1. IR spectra of crosslinked membranes before (A) and after (B) PLA removal. The peak at $1750 \mathrm{~cm}^{-1}$ is indicative of carbonyl groups from PLA and is nearly absent after treatment with aqueous base. The peaks at $970 \mathrm{~cm}^{-1}$ correspond to trans double bonds in polyDCPD, and after curing the film residue double bonds were not affected. 


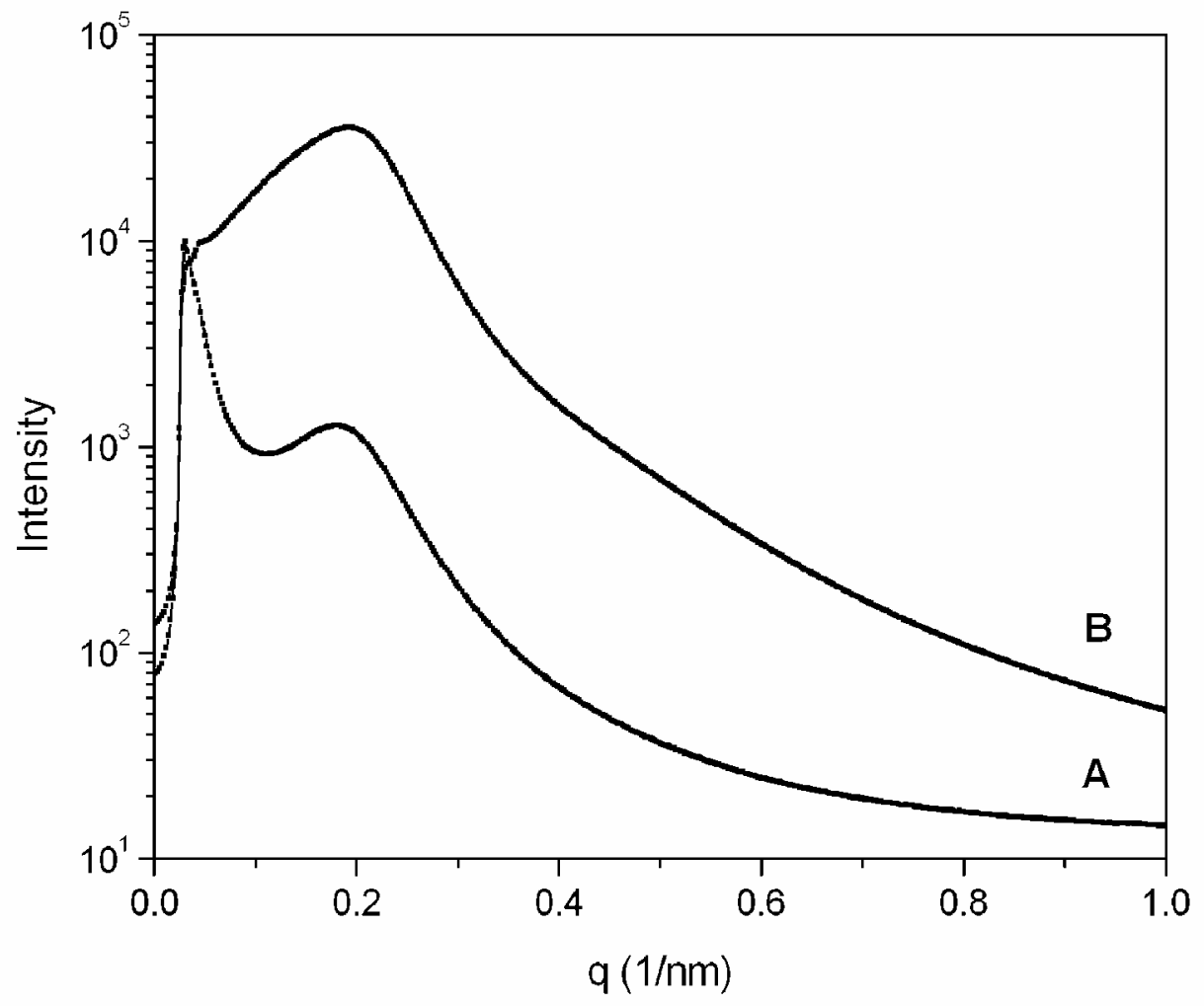

Figure S2. Synchrotron SAXS profiles of crosslinked membranes before (A) and after (B) PLA etching. The fixed peak position at $0.19 \mathrm{~nm}^{-1}$ suggests a domain spacing (D) of $33 \mathrm{~nm}$. The "strong upturn" at low values of q is actually for the sample before removal of the polylactide and is an instrumental artifact due to the beam stop in the SAXS instrument. A pore volume fraction in membranes is 0.41 based on the mass loss after PLA degradation that consists with the initial PLA composition. With the same pore volume fraction, for a hexagonally-packed cylindrical morphology, a calculated PLA domain size $(d)$ is about $22.2 \mathrm{~nm}\left(\pi d^{2} / 2 \sqrt{3} D^{2}=0.41\right)$; $d$ is $13.5 \mathrm{~nm}$ for a lamellar morphology $(\mathrm{D} \times 0.41)$. Herein, for a bicontinuous morphology, PLA domain size (or pore size) is presumably between these two values. After PLA degradation, enhancement in the scattered intensity is 28 times. A simple calculation is given below based on the fact that scattered intensity is proportional to the square of the electron density difference between two phases. The total electron density for PS is $0.560 \mathrm{~mol} \mathrm{e}^{-} \mathrm{cm}^{-3}$, for PLA $0.665 \mathrm{~mol}$

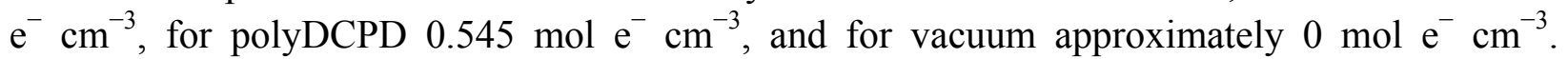
Therefore, the expected enhancement in scattering intensity is $(0.550-0)^{2} /(0.55-0.665)^{2}=23$, which agrees with the experimental value. 


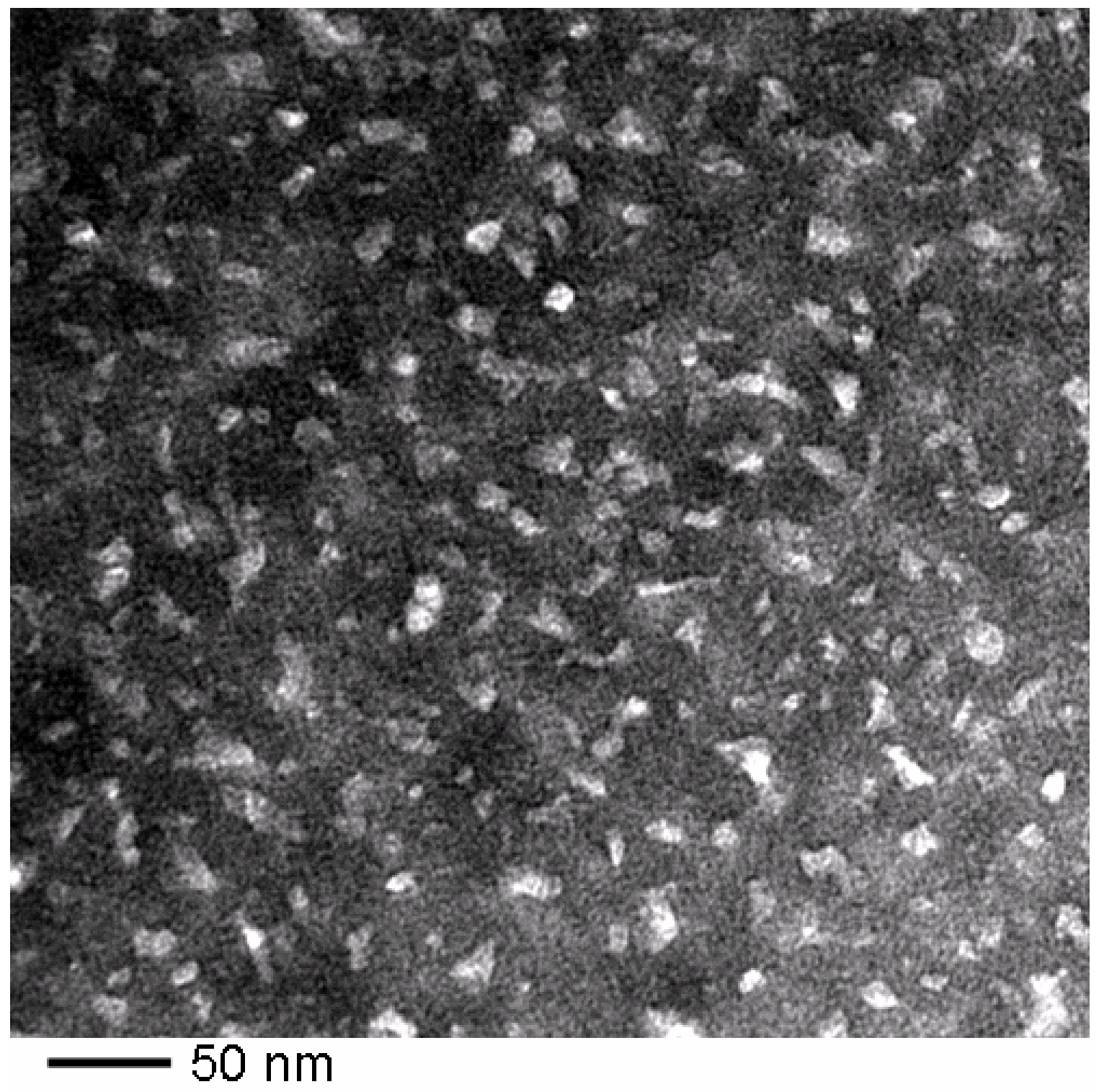

Figure S3. TEM image of the crosslinked membrane before PLA degradation. Bring regions correspond to PLA domains which are not stained by $\mathrm{OsO}_{4}$. Roughly, PLA domain size is in the range of 10 to $20 \mathrm{~nm}$, consistent with what we deduced from the SAXS experiment. 


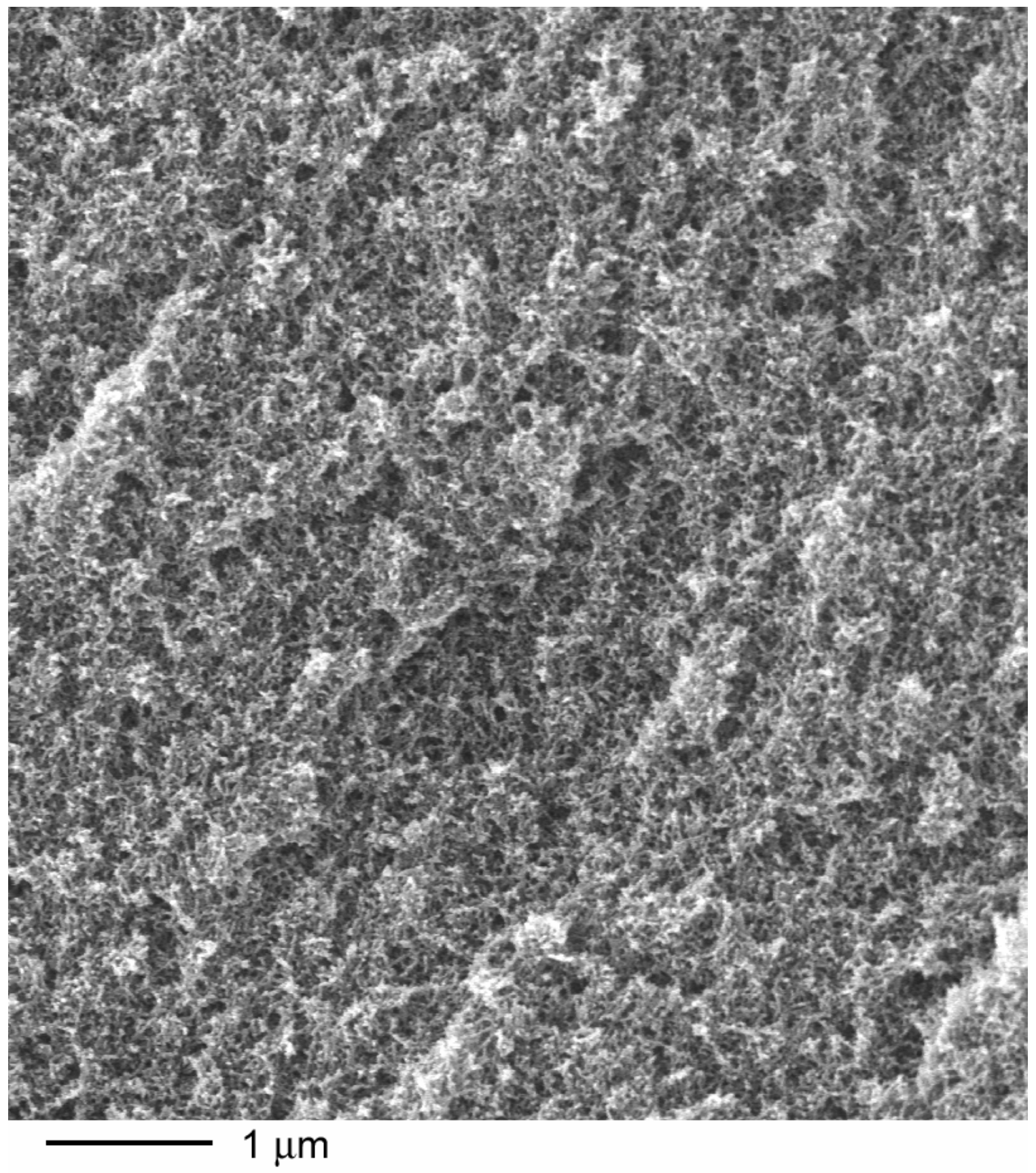

Figure S4. SEM image of the fractured surface of mesoporous polymer membrane. The porous membrane was fractured after freezing in liquid $\mathrm{N}_{2}$ and coated with $1.5 \mathrm{~nm} \mathrm{Pt}$. 


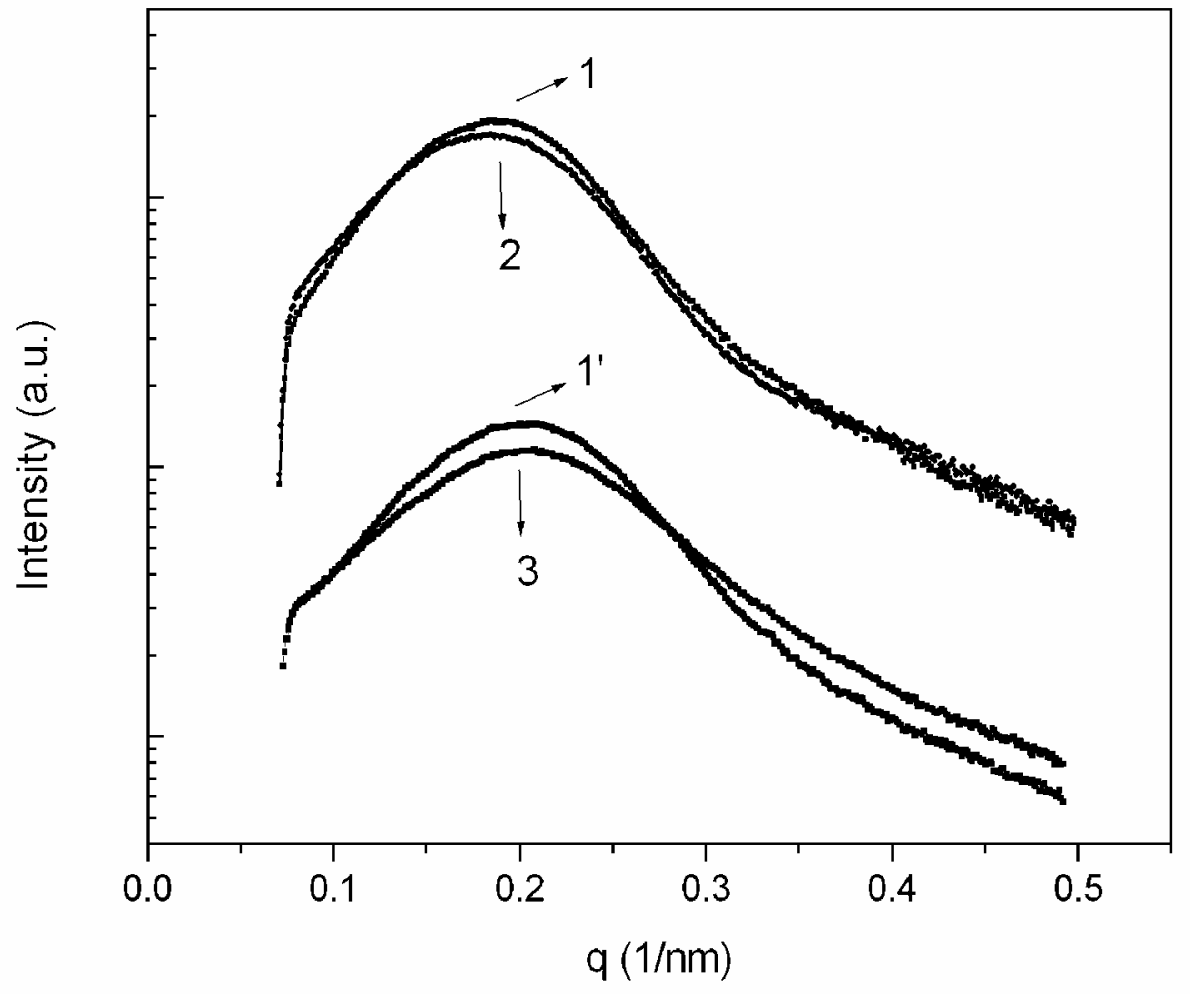

(a)

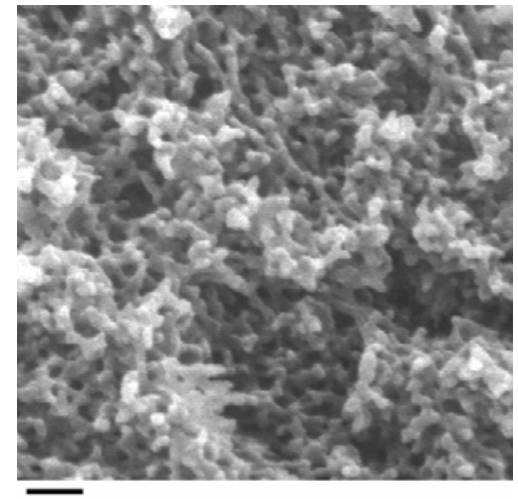

1

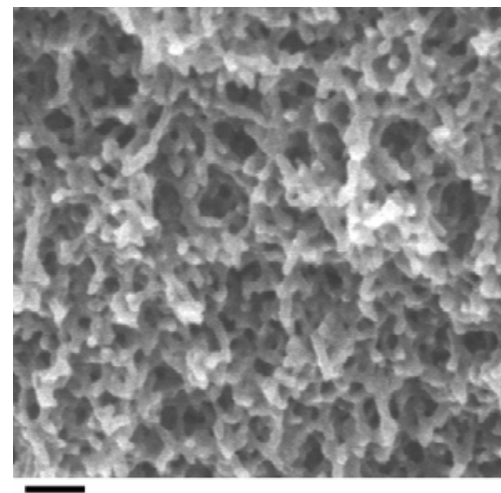

2

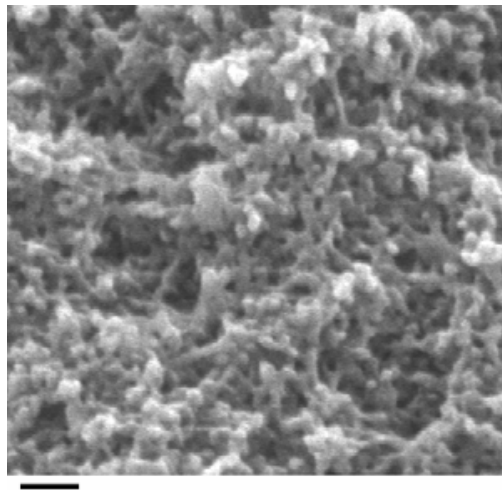

3

(b)

Figure S5. (a) SAXS profiles of crosslinked membranes after PLA etching at RT: 1(1'). no annealing; 2. after annealing at $140{ }^{\circ} \mathrm{C}$ for $1 \mathrm{~h}$; 3 . dried from THF (after soaking in THF, slow solvent-exchange with $\mathrm{MeOH}$ was performed before drying). (b) SEM images corresponding to freeze-fractured sample 1, 2, and 3 (scale bars above are $100 \mathrm{~nm}$ ). 


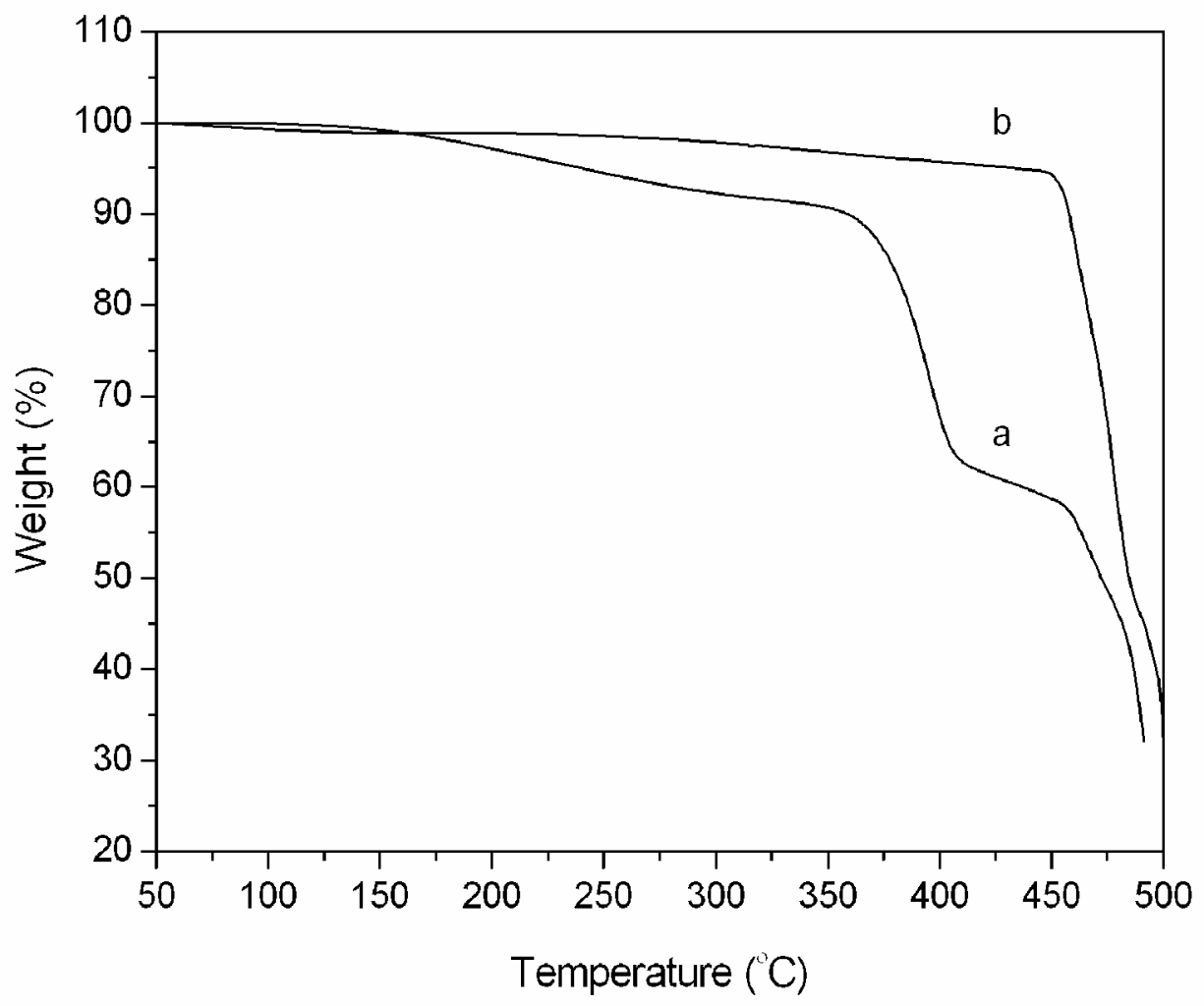

Figure S6. Thermogravimetric analysis of crosslinked membranes before (a) and after (b) PLA removal under air (heating rate: $20^{\circ} \mathrm{C} / \mathrm{min}$ ). 


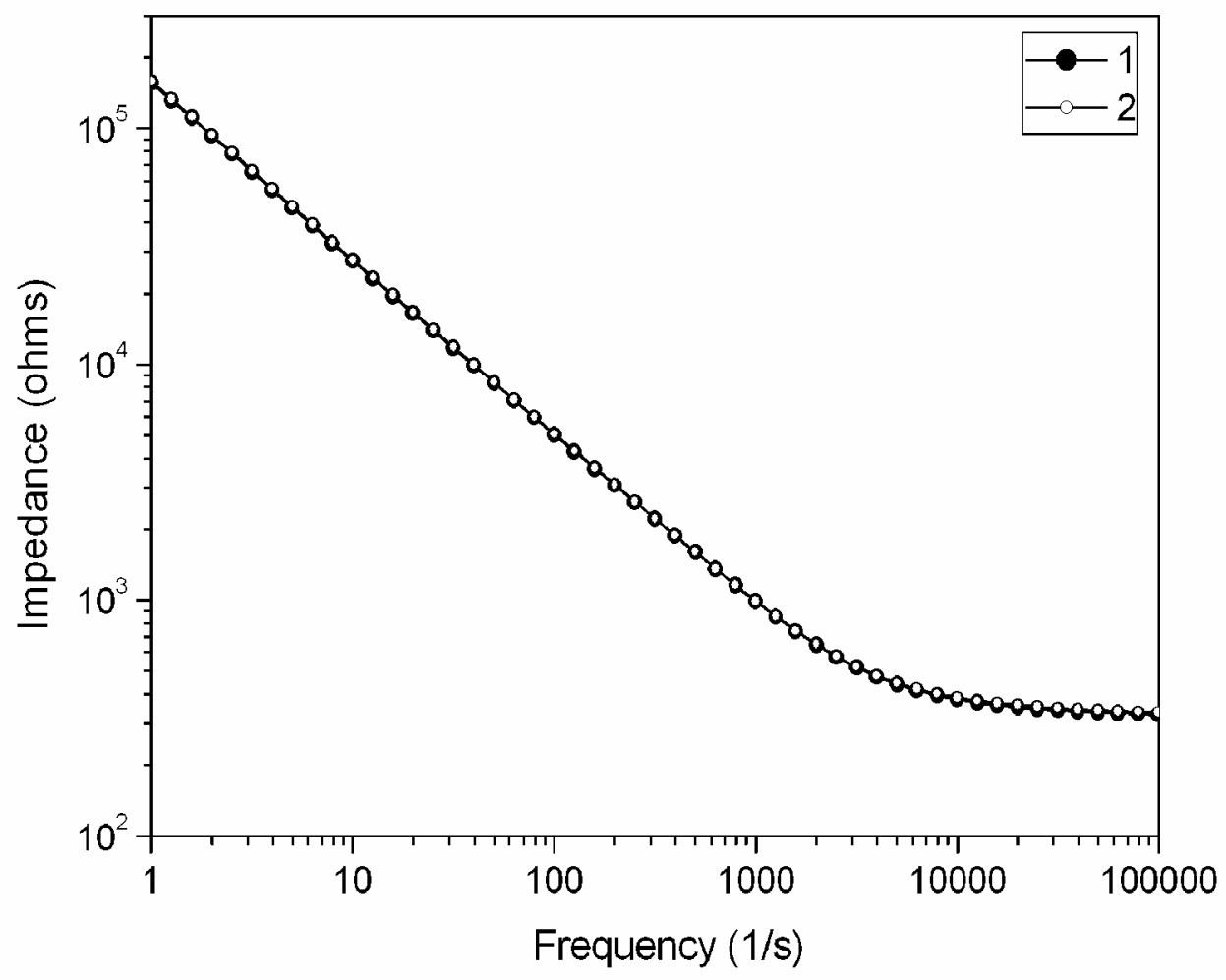

Figure S7. Conductivity measurement of the mesoporous membrane filled with the ionic liquid (IL) 1-ethyl-3-methylimidazolium bis(trifluoromethylsulfonyl)imide ([Emim][TFSI]) at $25^{\circ} \mathrm{C}$ (Trace 1 and 2 were two measurements on the same membrane). Conductivity of pure IL at 25 ${ }^{\circ} \mathrm{C}\left(\mathrm{I}_{1}\right)$ is $9.0 \times 10^{-3} \mathrm{~S} \mathrm{~cm}^{-1}$. This IL-filled membrane (size: $3.6 \times 3.0 \times 0.51 \mathrm{~mm}$ ) has an impedance of $335 \mathrm{ohms}$, and ionic conductivity $\left(\mathrm{I}_{2}\right)$ is $1.49 \times 10^{-3} \mathrm{~S} \mathrm{~cm}^{-1}$ (1.058×cell constant/resistance). Based on the pore volume fraction of 0.41 (Figure $\mathrm{S} 2$ ), a tortuosity is $2.48\left(\mathrm{I}_{1} \times 0.41 / \mathrm{I}_{2}\right)$. 


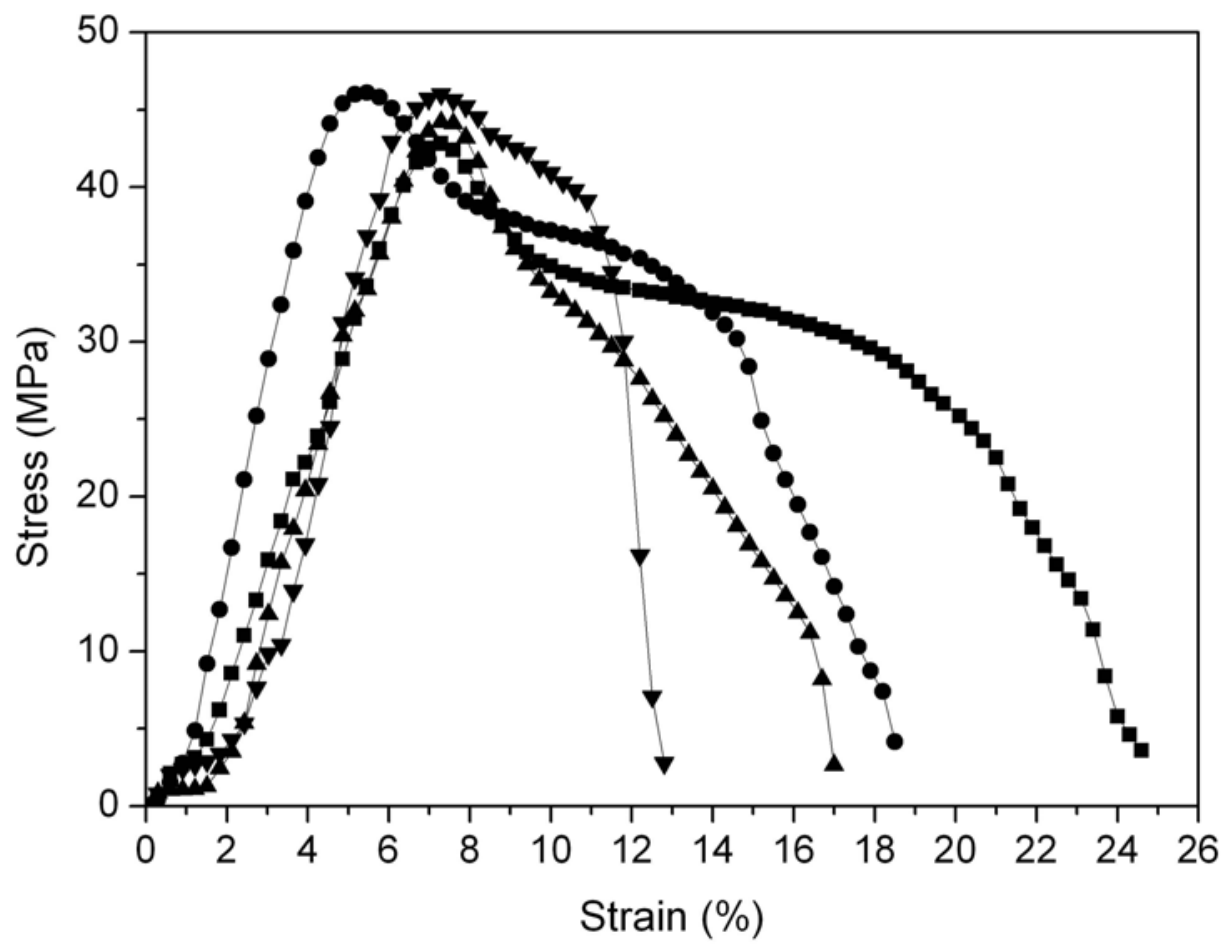

Figure S8. Tensile tests on crosslinked membranes before removing PLA. Defects from cutting membranes into a dogbone shape caused deviations in tensile tests; the averaged tensile strength is approximately $45 \mathrm{MPa}$ with the average elongation at break around $15 \%$. 


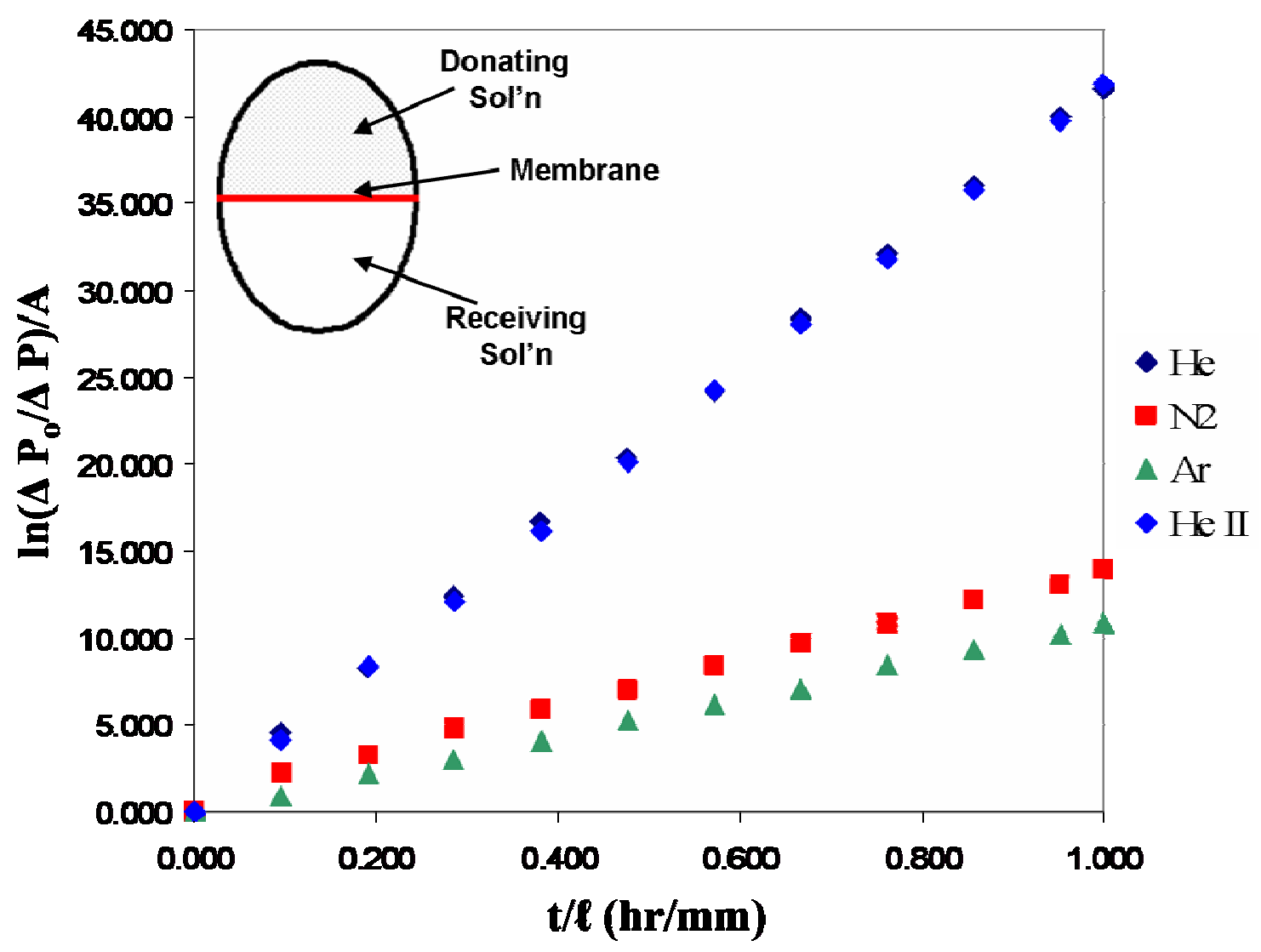

Figure S9. Diffusion data for different gases. As expected, effective diffusion coefficients are higher for gases with lower molecular weights. The two Helium runs show the typical reproducibility between samples.

The plot above was generated using the experimental procedure set forth by reference $9 \mathrm{c}$ in the paper. A membrane is placed in the gas diffusion cell to separate the donating and receiving volumes, as shown by the inset of the plot. The donating volume is filled with a high pressure charge of the gas to be studied to start the experiment. Pressure and temperature data are then recorded as a function of time for each volume and plotted as suggested by Equation 1.

$$
\frac{\Delta p_{o}}{\Delta p}=\exp \left[\frac{D_{e f f} A t}{\ell}\left(\frac{1}{V^{\prime}}+\frac{1}{V^{\prime \prime}}\right)\right]
$$

This allows the effective diffusion coefficient, $D_{\text {eff; }}$, to be calculated by knowing $\ell$ the membrane thickness, $A$ the membrane area, $t$ the time, $V$ ' and $V$ " the receiving and donating compartment volumes and $\Delta p_{o}$ and $\Delta p$ the pressure difference across the membrane initially and at time $t$. We then expect the diffusion coefficient inside the pore, $D$, to be related to $D_{\text {eff }}$ through the tortuosity, $\tau$, and porosity, $\varepsilon$, of the membrane as shown in Equation 2. 


$$
D=\frac{D_{e f f} \tau}{\varepsilon}
$$

A prediction for the diffusion coefficient can be made using a simple theory for Knudsen diffusion $^{3}$ which relies on the pore diameter, $d$, Boltzman's constant, $k_{B}$, temperature, $T$, and the mass of the gas molecule, $\tilde{m}$ :

$$
D_{K n}=\frac{d}{3}\left[\frac{2 k_{B} T}{\tilde{m}}\right]^{1 / 2}
$$

These predictions are compared with the experimental values in Table I. Table I also contains the values predicted for bulk diffusion coefficients by the Chapman-Enskog kinetic theory of gases. ${ }^{3}$ The close agreements between the experimental and Knudsen coefficients show that the transport through the pores occurs by a Knudsen mechanism. Knudsen diffusion is the result of collisions between the gas molecules and pore wall as opposed to intermolecular collisions between the gas molecules, which is the case in kinetic theory diffusion. This is the expected result because the pore diameter, $23 \mathrm{~nm}$, is about 10 times smaller than estimates of the gases' mean free path. Finally, Table I presents the gas selectivity relative to Helium as calculated through experiments and Knudsen theory.

$$
\alpha_{i, \exp }=D_{e f f, H e} / D_{e f f, i} \quad \alpha_{i, K n}=\sqrt{\tilde{m}_{H e} / \tilde{m}_{i}}
$$

Table I: Experimental and Predicted Diffusion Coefficients and Selectivities

\begin{tabular}{|c|c|c|c|c|c|c|}
\hline Solute & $\mathbf{D}_{\text {eff }}$ & $\mathbf{D}^{\mathbf{a}}$ & $\mathbf{D}_{\mathbf{K n}}^{\mathbf{b}}$ & $\mathbf{D}_{\mathbf{C - E}}$ & $\boldsymbol{\alpha}_{\exp }$ & $\boldsymbol{\alpha}_{\mathbf{K n}}$ \\
\hline $\mathrm{He}$ & 0.0154 & 0.089 & 0.085 & 1.1184 & 1.00 & 1.00 \\
\hline $\mathrm{N}_{2}$ & 0.0055 & 0.031 & 0.032 & 0.1350 & 2.82 & 2.66 \\
\hline $\mathrm{Ar}$ & 0.0047 & 0.027 & 0.027 & 0.1219 & 3.27 & 3.15 \\
\hline
\end{tabular}

Effective, Knudsen and kinetic theory diffusion coefficients have units of $\mathrm{cm}^{2} \mathrm{sec}^{-1}$.

a) Assuming a porosity of 0.40 and a tortuosity of 2.29.

b) Assuming a pore diameter of $23.0 \mathrm{~nm}$.

3 Cussler, E. L. Diffusion, 2nd ed.; Cambridge University Press: Cambridge, 2002. 